\title{
Existence results for fractional differential equations of arbitrary order with nonlocal integral boundary conditions
}

\author{
Bashir Ahmad ${ }^{1 *}$, Mohammed M Matar ${ }^{2}$ and Ravi P Agarwal ${ }^{1,3}$
}

\author{
"Correspondence: \\ bashirahmad_qau@yahoo.com \\ ${ }^{1}$ NAAM-Research Group, \\ Department of Mathematics, \\ Faculty of Science, King Abdulaziz \\ University, P.O. Box 80203, Jeddah, \\ 21589, Saudi Arabia \\ Full list of author information is \\ available at the end of the article
}

\begin{abstract}
In this paper, we investigate the existence of solutions for fractional differential equations of arbitrary order with nonlocal integral boundary conditions. The existence results are obtained by applying Krasnoselskii's fixed point theorem and Leray-Schauder degree theory, while the uniqueness of the solutions is established by means of Banach's contraction mapping principle. The paper concludes with illustrative examples.
\end{abstract}

MSC: 34A12; 34G20; 26A33

Keywords: fractional derivative; arbitrary order; integral boundary conditions; existence; fixed point

\section{Introduction}

The study of boundary value problems of fractional differential equations has gained considerable attention and several interesting results involving a variety of boundary conditions have appeared in the recent literature on the topic. The tools of fractional calculus have been effectively employed to improve the mathematical modeling of several phenomena occurring in scientific and engineering disciplines such as viscoelasticity [1], electrochemistry [2], electromagnetism [3], biology [4,5], optimal control [6, 7], diffusion process [8-10], economics [11], chaotic theory [12], variational problems [13], etc. For a theoretical development of the subject, concerning the existence and uniqueness of solutions for nonlinear fractional order initial and nonlocal boundary value problems, we refer the reader to [14-26] and the references cited therein. There has also been a great emphasis on studying fractional differential equations supplemented with integral boundary conditions; for instance, see [27-32]. Motivated by recent studies of nonlocal nonlinear integral boundary value problems of fractional order, we go a step further and consider a more general problem of nonlinear fractional differential equations of arbitrary order with nonlocal integral boundary conditions. Precisely, we investigate the following problem:

$$
\left\{\begin{array}{l}
{ }^{C} D_{t_{0}}^{\alpha} x(t)=f(t, x(t)), \quad t \in J, \\
x^{(k)}(\theta)=b_{k}+\int_{t_{0}}^{\theta} g_{k}(s, x(s)) d s, \quad k=0,1,2, \ldots, n-1, \theta \in J,
\end{array}\right.
$$

(c) 2015 Ahmad et al. This article is distributed under the terms of the Creative Commons Attribution 4.0 International License (http://creativecommons.org/licenses/by/4.0/), which permits unrestricted use, distribution, and reproduction in any medium, provided you give appropriate credit to the original author(s) and the source, provide a link to the Creative Commons license, and indicate if changes were made. 
where $J=\left[t_{0}, T\right],{ }^{C} D_{t_{0}}^{\alpha}$ is the Caputo fractional derivative of order $\alpha, n=[\alpha]+1, b_{k} \in \mathbb{R}$, and $f, g_{k}: J \times \mathbb{R} \rightarrow \mathbb{R}$ are given continuous functions.

The paper is organized as follows. In Section 2, we outline some basic concepts of fractional calculus, and establish a lemma which plays a key role in the sequel. The main results dealing with the existence and uniqueness of solutions for the problem (1.1) are discussed in Sections 3 and 4. We make use of the standard tools of the fixed point theory to obtain the desired results. The paper concludes with some interesting observations.

\section{Preliminaries}

First of all, we fix our terminology and recall some basic ideas of fractional calculus [14].

Let $C(J, \mathbb{R})$ be a Banach space of all continuous real valued functions defined on $J$ endowed with the norm defined by $\|x\|=\sup \{|x(t)|, t \in J\}$.

Definition 2.1 The Riemann-Liouville fractional integral of order $r$ for a function $h \in$ $C(J, \mathbb{R})$ is defined as

$$
I^{r} h(t)=\frac{1}{\Gamma(r)} \int_{t_{0}}^{t} \frac{h(s)}{(t-s)^{1-r}} d s, \quad r>0,
$$

provided the integral exists.

Definition 2.2 Let $h:\left[t_{0}, \infty\right) \rightarrow \mathbb{R}$ be such that $h \in A C^{n}(J, \mathbb{R})$. Then the Caputo derivative of fractional order $r$ for $h$ is defined as

$$
{ }^{c} D_{t_{0}}^{r} h(t)=\frac{1}{\Gamma(n-r)} \int_{t_{0}}^{t}(t-s)^{n-r-1} h^{(n)}(s) d s, \quad n-1<r<n, n=[r]+1,
$$

where $[r]$ denotes the integer part of the real number $r$.

Lemma 2.3 Let $x \in A C^{n}(J, \mathbb{R}), f \in A C(J, \mathbb{R})$, and $c_{k} \in \mathbb{R}$, then

$$
\left\{\begin{array}{l}
{ }^{C} D_{t_{0}}^{\alpha}\left(I^{\alpha} f(t)\right)=f(t), \\
I^{\alpha}\left({ }^{C} D_{t_{0}}^{\alpha} x(t)\right)=x(t)+c_{0}+c_{1}\left(t-t_{0}\right)+c_{2}\left(t-t_{0}\right)^{2}+\cdots+c_{n-1}\left(t-t_{0}\right)^{n-1} . \\
\text { The general solution of the equation }{ }^{C} D_{t_{0}}^{\alpha} x(t)=0 \text { is } \\
\quad x(t)=c_{0}+c_{1}\left(t-t_{0}\right)+c_{2}\left(t-t_{0}\right)^{2}+\cdots+c_{n-1}\left(t-t_{0}\right)^{n-1} .
\end{array}\right.
$$

We need the following result in the sequel.

Lemma 2.4 Let $\left\{u_{n}\right\}$ be a sequence of real numbers, and $n, k \in \mathbb{N}$, such that $0 \leq k \leq n-1$. If $v$ is a positive real number, then

$$
\sum_{m=0}^{n-k-1} \sum_{r=0}^{n-k-m-1}(-1)^{r} \frac{v^{r+m}}{r ! m !} u_{m+k+r}=u_{k}
$$

Proof The left-hand side of equation (2.1) can be rearranged as

$$
\sum_{m=0}^{n-k-1}\left(\sum_{r=0}^{m} \frac{(-1)^{m-r}}{r !(m-r) !}\right) v^{m} u_{k+m} .
$$


By a binomial expansion, the inner sum takes the form

$$
\frac{1}{m !} \sum_{r=0}^{m}(-1)^{m-r}\left(\begin{array}{c}
m \\
r
\end{array}\right)=0,
$$

for all $m \geq 1$. This completes the proof.

\section{Associated linear problem}

In this section, we consider the linear variant of the problem (1.1) given by

$$
\begin{aligned}
& { }^{C} D_{t_{0}}^{\alpha} x(t)=\tilde{f}(t), \quad t \in J, \\
& x^{(k)}(\theta)=b_{k}+\int_{t_{0}}^{\theta} g_{k}(s) d s, \quad k=0,1,2, \ldots, n-1, \theta \in J,
\end{aligned}
$$

where $x \in A C^{n}(J, \mathbb{R}), \tilde{f} \in A C(J, \mathbb{R})$ and $g_{k} \in C(J, \mathbb{R})$.

Lemma 3.1 The fractional boundary value problem (3.1) is equivalent to the integral equation

$$
x(t)=\sum_{k=0}^{n-1} \frac{(t-\theta)^{k}}{k !}\left(b_{k}+\int_{t_{0}}^{\theta} g_{k}(s) d s-I^{\alpha-k} \tilde{f}(\theta)\right)+I^{\alpha} \tilde{f}(t), \quad t \in J .
$$

Proof For $\alpha=n$, problem (3.1) reduces to the $n$th order classical problem:

$$
\begin{aligned}
& \frac{d^{n}}{d t^{n}} x(t)=\tilde{f}(t), \quad t \in J, \\
& x^{(k)}(\theta)=b_{k}+\int_{t_{0}}^{\theta} g_{k}(s) d s, \quad k=0,1,2, \ldots, n-1, \theta \in J,
\end{aligned}
$$

which can be integrated $n$ times to have

$$
x(t)=\sum_{k=0}^{n-1} \frac{(t-\theta)^{k}}{k !}\left(b_{k}+\int_{t_{0}}^{\theta} g_{k}(s) d s\right)+\frac{1}{(n-1) !} \int_{\theta}^{t}(t-s)^{n-1} \tilde{f}(s) d s .
$$

Using binomial expansion, we find that

$$
\begin{aligned}
& \frac{1}{(n-1) !} \int_{\theta}^{t}(t-s)^{n-1} \tilde{f}(s) d s \\
& =\frac{1}{(n-1) !} \int_{t_{0}}^{t}(t-s)^{n-1} \tilde{f}(s) d s-\frac{1}{(n-1) !} \int_{t_{0}}^{\theta}(t-s)^{n-1} \tilde{f}(s) d s \\
& =I^{n} \tilde{f}(t)-\frac{1}{(n-1) !} \int_{t_{0}}^{\theta}(t-\theta+\theta-s)^{n-1} \tilde{f}(s) d s \\
& =I^{n} \tilde{f}(t)-\sum_{k=0}^{n-1} \frac{(t-\theta)^{k}}{k !}\left(\frac{1}{(n-k-1) !} \int_{t_{0}}^{\theta}(\theta-s)^{n-k-1} \tilde{f}(s) d s\right) \\
& =I^{n} \tilde{f}(t)-\sum_{k=0}^{n-1} \frac{(t-\theta)^{k}}{k !} I^{n-k} \tilde{f}(\theta),
\end{aligned}
$$


which together with (3.3) yields (3.2). Next, for $n-1<\alpha<n$, by Lemma 2.3, we have

$$
I^{\alpha} \tilde{f}(t)=I^{\alpha}\left({ }^{C} D_{t_{0}}^{\alpha}\right) x(t)=x(t)+\sum_{k=0}^{n-1} c_{k}\left(t-t_{0}\right)^{k} .
$$

Differentiating (3.4) $k$ times, we get

$$
\sum_{m=0}^{n-k-1} \frac{(k+m) !}{m !} c_{m+k}\left(t-t_{0}\right)^{m}=I^{\alpha-k} \tilde{f}(t)-x^{(k)}(t)
$$

for $0 \leq k \leq n-1$. In view of the integral boundary conditions in (3.1), we can express equation (3.5) in the form of the following array:

$$
\begin{aligned}
& {\left[\begin{array}{cccccc}
1 & \frac{1 !}{1 !}\left(\theta-t_{0}\right)^{1} & \frac{2 !}{2 !}\left(\theta-t_{0}\right)^{2} & \ldots & \frac{(n-2) !}{(n-2) !}\left(\theta-t_{0}\right)^{n-2} & \frac{(n-1) !}{(n-1) !}\left(\theta-t_{0}\right)^{n-1} \\
0 & \frac{1}{0 !}\left(\theta-t_{0}\right)^{0} & \frac{2 !}{1 !}\left(\theta-t_{0}\right)^{1} & \ldots & \frac{(n-2) !}{(n-3) !}\left(\theta-t_{0}\right)^{n-3} & \frac{(n-1) !}{(n-2) !}\left(\theta-t_{0}\right)^{n-2} \\
0 & 0 & \frac{2 !}{0 !}\left(\theta-t_{0}\right)^{0} & \ldots & \frac{(n-2) !}{(n-4) !}\left(\theta-t_{0}\right)^{n-4} & \frac{(n-1) !}{(n-3) !}\left(\theta-t_{0}\right)^{n-3} \\
\vdots & \vdots & \vdots & \ldots & \vdots & \vdots \\
0 & 0 & 0 & 0 & \frac{(n-2) !}{0 !}\left(\theta-t_{0}\right)^{0} & \frac{(n-1) !}{1 !}\left(\theta-t_{0}\right)^{1} \\
0 & 0 & 0 & 0 & 0 & \frac{(n-1) !}{0 !}\left(\theta-t_{0}\right)^{0}
\end{array}\right]} \\
& \times\left[\begin{array}{c}
c_{0} \\
c_{1} \\
c_{2} \\
\vdots \\
c_{n-2} \\
c_{n-1}
\end{array}\right]=\left[\begin{array}{c}
I^{\alpha} \tilde{f}(\theta)-b_{0}-\int_{t_{0}}^{\theta} g_{0}(s) d s \\
I^{\alpha-1} \tilde{f}(\theta)-b_{1}-\int_{t_{0}}^{\theta} g_{1}(s) d s \\
I^{\alpha-2} \tilde{f}(\theta)-b_{2}-\int_{t_{0}}^{\theta} g_{2}(s) d s \\
\vdots \\
I^{\alpha-n+2} \tilde{f}(\theta)-b_{n-2}-\int_{t_{0}}^{\theta} g_{n-2}(s) d s \\
I^{\alpha-n+1} \tilde{f}(\theta)-b_{n-1}-\int_{t_{0}}^{\theta} g_{n-1}(s) d s
\end{array}\right] .
\end{aligned}
$$

Solving this system for $c_{r}, r=1,2, \ldots, n$, we obtain

$$
c_{n-r}=\frac{1}{(n-r) !} \sum_{k=0}^{r-1} \frac{(-1)^{k}\left(\theta-t_{0}\right)^{k}}{k !}\left(I^{\alpha-n+r-k} \tilde{f}(\theta)-b_{n-r+k}-\int_{t_{0}}^{\theta} g_{n-r+k}(s) d s\right),
$$

which, for $m=0,1,2, \ldots, n-1$, can be written as

$$
c_{m}=\frac{1}{m !} \sum_{k=0}^{n-m-1} \frac{(-1)^{k}\left(\theta-t_{0}\right)^{k}}{k !}\left(I^{\alpha-m-k} \tilde{f}(\theta)-b_{m+k}-\int_{t_{0}}^{\theta} g_{m+k}(s) d s\right) .
$$

Indeed, by Lemma 2.4 with $v=\theta-t_{0}$, and $u_{k}=I^{\alpha-k} \tilde{f}(\theta)-b_{k}-\int_{t_{0}}^{\theta} g_{k}(s) d s$, (3.6) is a solution of (3.5) with $t=\theta$. By means of (3.6) and (3.4), we obtain

$$
x(t)=I^{\alpha} \tilde{f}(t)+\sum_{k=0}^{n-1} \sum_{m=0}^{n-k-1} \frac{(-1)^{m}\left(\theta-t_{0}\right)^{m}\left(t-t_{0}\right)^{k}}{m ! k !}\left(b_{m+k}+\int_{t_{0}}^{\theta} g_{m+k}(s) d s-I^{\alpha-k-m} \tilde{f}(\theta)\right),
$$

which can alternatively be written as

$$
x(t)=I^{\alpha} \tilde{f}(t)+\sum_{k=0}^{n-1} \psi_{k}(t)\left(b_{k}+\int_{t_{0}}^{\theta} g_{k}(s) d s-I^{\alpha-k} f(\theta)\right)
$$


where

$$
\psi_{k}(t)=\sum_{m=0}^{k}(-1)^{k-m} \frac{\left(\theta-t_{0}\right)^{k-m}}{(k-m) !} \frac{\left(t-t_{0}\right)^{m}}{m !}, \quad k=0,1, \ldots, n-1 .
$$

Next, for $t_{0}<\theta \leq T$, it follows by a binomial expansion that

$$
\psi_{k}(t)=\frac{\left(\theta-t_{0}\right)^{k}}{k !} \sum_{m=0}^{k}\left(\begin{array}{l}
k \\
m
\end{array}\right)\left(\frac{t-t_{0}}{\theta-t_{0}}\right)^{m}(-1)^{k-m}=\frac{\left(\theta-t_{0}\right)^{k}}{k !}\left(\frac{t-\theta}{\theta-t_{0}}\right)^{k}=\frac{(t-\theta)^{k}}{k !},
$$

which, on substituting in (3.7), yields (3.2).

On the other hand, applying the operator ${ }^{C} D_{t_{0}}^{\alpha}, n-1<\alpha \leq n$ to (3.2), and using Lemma 2.3, we obtain (3.1). This completes the proof.

Remark 3.2 We can solve different kinds of boundary value problems involving integral (classical) and multi-point boundary conditions by applying the method of proof used for Lemma 3.1. Here, we enlist the following two cases.

(a) The two-point boundary value problem of the form

$$
\left\{\begin{array}{l}
{ }^{C} D_{t_{0}}^{\alpha} x(t)=\tilde{f}(t), \quad t \in J, \\
x\left(t_{0}\right)=b_{0}, \quad x^{(k)}(T)=b_{k}+\int_{t_{0}}^{T} g_{k}(s) d s, \quad k=1,2, \ldots, n-1,
\end{array}\right.
$$

has an integral solution given by

$$
\begin{aligned}
x(t)= & b_{0}+\int_{t_{0}}^{t} \frac{(t-s)^{\alpha-1}}{\Gamma(\alpha)} \tilde{f}(s) d s-\sum_{k=1}^{n-1} \frac{(-1)^{k}}{k !}\left(\left(T-t_{0}\right)^{k}-(T-t)^{k}\right) \\
& \times\left(b_{k}+\int_{t_{0}}^{T} g_{k}(s) d s-\int_{t_{0}}^{T} \frac{(T-s)^{\alpha-k-1}}{\Gamma(\alpha-k)} \tilde{f}(s) d s\right), \quad t \in J .
\end{aligned}
$$

(b) The integral solution of the three-point boundary value problem

$$
\left\{\begin{array}{l}
{ }^{C} D_{t_{0}}^{\alpha} x(t)=\tilde{f}(t), \quad t \in J, \\
x\left(t_{0}\right)=b_{0}, \quad x\left(t_{1}\right)=b_{1}, \quad t_{0}<t_{1}<T \\
x^{(k)}(T)=b_{k}+\int_{t_{0}}^{T} g_{k}(s) d s, \quad k=2, \ldots, n-1
\end{array}\right.
$$

is

$$
\begin{aligned}
x(t)= & \int_{t_{0}}^{t} \frac{(t-s)^{\alpha-1}}{\Gamma(\alpha)} \tilde{f}(s) d s+b_{0}-\left(b_{0}-b_{1}+\int_{t_{0}}^{t_{1}} \frac{\left(t_{1}-s\right)^{\alpha-1}}{\Gamma(\alpha)} f(s) d s\right) \frac{t-t_{0}}{t_{1}-t_{0}} \\
& +\sum_{k=2}^{n-1} \frac{(-1)^{k}}{k !}\left[\left(t-t_{0}\right)\left(T-t_{1}\right)^{k}-\left(t-t_{1}\right)\left(T-t_{0}\right)^{k}-\left(t_{1}-t_{0}\right)(T-t)^{k}\right] \\
& \times \frac{1}{t_{1}-t_{0}}\left(b_{k}+\int_{t_{0}}^{T} g_{k}(s) d s-\int_{t_{0}}^{T} \frac{(T-s)^{\alpha-k-1}}{\Gamma(\alpha-k)} \tilde{f}(s) d s\right), \quad t \in J .
\end{aligned}
$$

4 Main results

In this section, we show the existence of solutions for the problem (1.1) by applying some fixed point theorems. 
In relation to the problem (1.1), we define the fixed point problem

$$
\Psi x=x,
$$

where the operator $\Psi: C(J, \mathbb{R}) \rightarrow C(J, \mathbb{R})$ is defined by

$$
\begin{aligned}
\Psi x(t)= & \sum_{k=0}^{n-1} \frac{(t-\theta)^{k}}{k !}\left(b_{k}+\int_{t_{0}}^{\theta} g_{k}(s, x(s))-\int_{t_{0}}^{\theta} \frac{(\theta-s)^{\alpha-k-1}}{\Gamma(\alpha-k)} f(s, x(s)) d s\right) \\
& +\int_{t_{0}}^{t} \frac{(t-s)^{\alpha-1}}{\Gamma(\alpha)} f(s, x(s)) d s .
\end{aligned}
$$

Observe that the problem (1.1) has solutions if the operator equation (4.1) has fixed points.

Lemma 4.1 The operator $\Psi: C(J, \mathbb{R}) \rightarrow C(J, \mathbb{R})$ given by (4.2) is completely continuous.

Proof Obviously continuity of the operator $\Psi$ follows from the continuity of the functions $f$ and $g_{k}, k=0,1, \ldots, n-1$. Let $\Omega$ be a bounded subset of $C(J, \mathbb{R})$, then for any $t \in J$, and $x \in \Omega$, there exist positive constants $L_{f}$, and $L_{k}, k=0,1, \ldots, n-1$, such that $|f(t, x(t))| \leq L_{f}$, and $\left|g_{k}(t, x(t))\right| \leq L_{k}$. Then we have

$$
\begin{aligned}
& |(\Psi x)(t)| \\
& \leq \sum_{k=0}^{n-1} \frac{|t-\theta|^{k}}{k !}\left(\left|b_{k}\right|+\int_{t_{0}}^{\theta}\left|g_{k}(s, x(s))\right| d s+\int_{t_{0}}^{\theta} \frac{(\theta-s)^{\alpha-k-1}}{\Gamma(\alpha-k)}|f(s, x(s))| d s\right) \\
& \quad+\int_{t_{0}}^{t} \frac{(t-s)^{\alpha-1}}{\Gamma(\alpha)}|f(s, x(s))| d s \\
& \leq \sum_{k=0}^{n-1} \frac{|t-\theta|^{k}}{k !}\left(\left|b_{k}\right|+\left(\theta-t_{0}\right) L_{k}+\frac{\left(\theta-t_{0}\right)^{\alpha-k}}{\Gamma(\alpha-k+1)} L_{f}\right)+\frac{\left(t-t_{0}\right)^{\alpha}}{\Gamma(\alpha+1)} L_{f} \\
& =: L,
\end{aligned}
$$

which implies that $\|(\Psi x)\| \leq L$. Furthermore,

$$
\begin{aligned}
& \left|(\Psi x)\left(t_{2}\right)-(\Psi x)\left(t_{1}\right)\right| \\
& \leq \sum_{k=0}^{n-1} \frac{\left|\left(t_{2}-\theta\right)^{k}-\left(t_{1}-\theta\right)^{k}\right|}{k !} \\
& \quad \times\left(\left|b_{k}\right|+\int_{t_{0}}^{\theta}\left|g_{k}(s, x(s))\right| d s+\int_{t_{0}}^{\theta} \frac{(\theta-s)^{\alpha-k-1}}{\Gamma(\alpha-k)}|f(s, x(s))| d s\right) \\
& \quad+\int_{t_{0}}^{t_{1}} \frac{\left|\left(t_{2}-s\right)^{\alpha-1}-\left(t_{1}-s\right)^{\alpha-1}\right|}{\Gamma(\alpha)}|f(s, x(s))| d s+\int_{t_{1}}^{t_{2}} \frac{\left(t_{2}-s\right)^{\alpha-1}}{\Gamma(\alpha)}|f(s, x(s))| d s \\
& \leq \sum_{k=0}^{n-1} \frac{\left|\left(t_{2}-\theta\right)^{k}-\left(t_{1}-\theta\right)^{k}\right|}{k !}\left(\left|b_{k}\right|+\left(\theta-t_{0}\right) L_{k}+\frac{\left(\theta-t_{0}\right)^{\alpha-k}}{\Gamma(\alpha-k+1)} L_{f}\right) \\
& \quad+\frac{L_{f}}{\Gamma(\alpha+1)}\left(2\left|t_{2}-t_{1}\right|^{\alpha}+\left|\left(t_{2}-t_{0}\right)^{\alpha}-\left(t_{1}-t_{0}\right)^{\alpha}\right|\right),
\end{aligned}
$$


which tends to zero independent of $x$ as $t_{2} \rightarrow t_{1}$. This implies that $\Psi$ is equicontinuous on $J$. In consequence, it follows by the Arzela-Ascoli theorem that the operator $\Psi$ is completely continuous.

Our first existence result is based on Krasnoselskii's fixed point theorem (4.2).

Theorem 4.2 ([33]) Let $M$ be a closed convex and nonempty subset of a Banach space $X$. Let $A, B$ be the operators such that

(i) $A x+B y \in M$ whenever $x, y \in M$;

(ii) $A$ is compact and continuous;

(iii) $B$ is a contraction.

Then there exists $z \in M$ such that $z=A z+B z$.

Theorem 4.3 Assume that

$\left(\mathrm{A}_{1}\right)$ For any $t \in J$ and $k=0,1, \ldots, n-1$, there exist positive constants $C_{f}$ and $C_{k}$ such that

$$
|f(t, x)-f(t, y)| \leq C_{f}|x-y|, \quad\left|g_{k}(t, x)-g_{k}(t, y)\right| \leq C_{k}|x-y|
$$

for all $x, y \in \mathbb{R}$, and we can find $\mu_{f}, \mu_{k} \in C\left(J, \mathbb{R}^{+}\right)$such that

$$
|f(t, x)| \leq \mu_{f}(t), \quad\left|g_{k}(t, x)\right| \leq \mu_{k}(t), \quad \text { for all } x \in \mathbb{R}
$$

$\left(\mathrm{A}_{2}\right) \eta<1$, where

$$
\eta=\sum_{k=0}^{n-1} \frac{(T-\theta)^{k}}{k !}\left[C_{k}\left(\theta-t_{0}\right)+\frac{C_{f}\left(\theta-t_{0}\right)^{\alpha-k}}{\Gamma(\alpha-k+1)}\right] .
$$

Then the problem (1.1) has at least one solution on J.

Proof Let us define a set $B_{r}=\{x \in C(J, \mathbb{R}):\|x\| \leq r\}$, where $r$ is a positive constant satisfying the inequality

$$
r \geq \frac{\left|T-t_{0}\right|^{\alpha}}{\Gamma(\alpha+1)}\left\|\mu_{f}\right\|+\sum_{k=0}^{n-1} \frac{|T-\theta|^{k}}{k !}\left(\left|b_{k}\right|+\left\|\mu_{k}\right\|+\left\|\mu_{f}\right\| \frac{\left(\theta-t_{0}\right)^{\alpha-k}}{\Gamma(\alpha-k+1)}\right) .
$$

Introduce the operators $\Theta$ and $\Phi$ on $B_{r}$ as

$$
\begin{aligned}
& (\Theta x)(t)=\int_{t_{0}}^{t} \frac{(t-s)^{\alpha-1}}{\Gamma(\alpha)} f(s, x(s)) d s, \\
& (\Phi x)(t)=\sum_{k=0}^{n-1} \frac{(t-\theta)^{k}}{k !}\left(b_{k}+\int_{t_{0}}^{\theta} g_{k}(s, x(s)) d s-\int_{t_{0}}^{\theta} \frac{(\theta-s)^{\alpha-k-1}}{\Gamma(\alpha-k)} f(s, x(s)) d s\right) .
\end{aligned}
$$

For $x, y \in B_{r}, t \in J$, using assumption $\left(\mathrm{A}_{1}\right)$, we find that

$$
\begin{aligned}
|\Theta x(t)+\Phi y(t)| \leq & \frac{\left(T-t_{0}\right)^{\alpha}}{\Gamma(\alpha+1)}\left\|\mu_{f}\right\| \\
& +\sum_{k=0}^{n-1} \frac{|T-\theta|^{k}}{k !}\left(\left|b_{k}\right|+\left\|\mu_{k}\right\|+\left\|\mu_{f}\right\| \frac{\left(\theta-t_{0}\right)^{\alpha-k}}{\Gamma(\alpha-k+1)}\right) \leq r .
\end{aligned}
$$


Thus, $\Theta x+\Phi y \in B_{r}$. By assumption $\left(\mathrm{A}_{1}\right)$, for $x, y \in B_{r}, t \in J$, we have

$$
\begin{aligned}
|(\Phi x)(t)-(\Phi y)(t)| & \leq \sum_{k=0}^{n-1} \frac{(T-\theta)^{k}}{k !}\left[C_{k}\left(\theta-t_{0}\right)+\frac{C_{f}\left(\theta-t_{0}\right)^{\alpha-k}}{\Gamma(\alpha-k+1)}\right]\|x-y\| \\
& \leq \eta\|x-y\|,
\end{aligned}
$$

that is, $\|(\Phi x)-(\Phi y)\| \leq \eta\|x-y\|$. Since $\eta<1$ by $\left(\mathrm{A}_{2}\right), \Phi$ is a contraction.

Continuity of $f$ implies that the operator $\Theta$ is continuous. Also, $\Theta$ is uniformly bounded on $B_{r}$ as

$$
\|\Theta x\| \leq \frac{\left(T-t_{0}\right)^{\alpha}}{\Gamma(\alpha+1)}\left\|\mu_{f}\right\|
$$

Now we show the compactness of the operator $\Theta$. In view of assumption $\left(A_{1}\right)$, we define

$$
\sup _{(t, x) \in J \times B r}|f(t, x)|=f_{\max }<\infty
$$

Then, for $t_{1}, t_{2} \in J$, we have

$$
\begin{aligned}
\left|(\Theta x)\left(t_{2}\right)-(\Theta x)\left(t_{1}\right)\right|= & \mid \int_{t_{0}}^{t_{1}} \frac{\left[\left(t_{2}-s\right)^{\alpha-1}-\left(t_{1}-s\right)^{\alpha-1}\right]}{\Gamma(\alpha)} f(s, x(s)) d s \\
& +\int_{t_{1}}^{t_{2}} \frac{\left(t_{2}-s\right)^{q-1}}{\Gamma(\alpha)} f(s, x(s)) d s \mid \\
\leq & \frac{f_{\max }}{\Gamma(\alpha+1)}\left(2\left|t_{2}-t_{1}\right|^{\alpha}+\left|\left(t_{2}-t_{0}\right)^{\alpha}-\left(t_{1}-t_{0}\right)^{\alpha}\right|\right),
\end{aligned}
$$

which is independent of $x$ and tends to zero as $t_{2} \rightarrow t_{1}$. So $\Theta$ is relatively compact on $B_{r}$. Hence, by the Arzela-Ascoli theorem, $\Theta$ is compact on $B_{r}$. Thus all the assumptions of Theorem 4.2 are satisfied. Therefore, the problem (1.1) has at least one solution on $J$. This completes the proof.

Our next result deals with the uniqueness of solutions for the problem (1.1) and is based on the contraction mapping principle due to Banach.

Theorem 4.4 Assume that $\left(\mathrm{A}_{1}\right)$ holds and that $\beta<1$, where

$$
\beta=\sum_{k=0}^{n-1} \frac{(T-\theta)^{k}}{k !}\left(C_{k}\left(\theta-t_{0}\right)+\frac{\left(\theta-t_{0}\right)^{\alpha-k}}{\Gamma(\alpha-k+1)} C_{f}\right)+\frac{\left(T-t_{0}\right)^{\alpha}}{\Gamma(\alpha+1)} C_{f}
$$

Then there exists a unique solution for the problem (1.1) on J.

Proof Setting $\sup _{t \in J}|f(t, 0)|=A_{f}$, $\sup _{t \in J}\left|g_{k}(t, 0)\right|=A_{k}$, and

$$
r \geq(1-\beta)^{-1}\left[\sum_{k=0}^{n-1} \frac{(T-\theta)^{k}}{k !}\left(\left|b_{k}\right|+A_{k}\left(\theta-t_{0}\right)+\frac{\left(\theta-t_{0}\right)^{\alpha-k}}{\Gamma(\alpha-k+1)} A_{f}\right)+\frac{\left(T-t_{0}\right)^{\alpha}}{\Gamma(\alpha+1)} A_{f}\right]
$$


we show that $\Psi B_{r} \subset B_{r}$, where $B_{r}=\{x \in C(J, \mathbb{R}):\|x\| \leq r\}$. For $x \in B_{r}$, we have

$$
\begin{aligned}
|\Psi x(t)| \leq & \sum_{k=0}^{n-1} \frac{|t-\theta|^{k}}{k !}\left(\left|b_{k}\right|+\int_{t_{0}}^{\theta}\left|g_{k}(s, x(s))\right| d s+\int_{t_{0}}^{\theta} \frac{(\theta-s)^{\alpha-k-1}}{\Gamma(\alpha-k)}|f(s, x(s))| d s\right) \\
& +\int_{t_{0}}^{t} \frac{(t-s)^{\alpha-1}}{\Gamma(\alpha)}|f(s, x(s))| d s \\
\leq & \sum_{k=0}^{n-1} \frac{|t-\theta|^{k}}{k !}\left(\left|b_{k}\right|+\int_{t_{0}}^{\theta}\left(\left|g_{k}(s, x(s))-g_{k}(s, 0)\right|+\left|g_{k}(s, 0)\right|\right) d s\right. \\
& \left.+\int_{t_{0}}^{\theta} \frac{(\theta-s)^{\alpha-k-1}}{\Gamma(\alpha-k)}(|f(s, x(s))-f(s, 0)|+|f(s, 0)|) d s\right) \\
& +\int_{t_{0}}^{t} \frac{(t-s)^{\alpha-1}}{\Gamma(\alpha)}(|f(s, x(s))-f(s, 0)|+|f(s, 0)|) d s \\
\leq & \sum_{k=0}^{n-1} \frac{|t-\theta|^{k}}{k !}\left(\left|b_{k}\right|+\left(\theta-t_{0}\right)\left(C_{k}\|x\|+A_{k}\right)\right. \\
& \left.+\frac{\left(\theta-t_{0}\right)^{\alpha-k}}{\Gamma(\alpha-k+1)}\left(C_{f}\|x\|+A_{f}\right)\right)+\frac{\left(t-t_{0}\right)^{\alpha}}{\Gamma(\alpha+1)}\left(C_{f}\|x\|+A_{f}\right) \\
\leq & \sum_{k=0}^{n-1} \frac{|T-\theta|^{k}}{k !}\left(\left|b_{k}\right|+A_{k}\left(\theta-t_{0}\right)+\frac{\left(\theta-t_{0}\right)^{\alpha-k}}{\Gamma(\alpha-k+1)} A_{f}\right)+\frac{\left(T-t_{0}\right)^{\alpha}}{\Gamma(\alpha+1)} A_{f} \\
& +\left(\sum_{k=0}^{n-1} \frac{|T-\theta|^{k}}{k !}\left(C_{k}\left(\theta-t_{0}\right)+\frac{\left(\theta-t_{0}\right)^{\alpha-k}}{\Gamma(\alpha-k+1)} C_{f}\right)+\frac{\left(T-t_{0}\right)^{\alpha}}{\Gamma(\alpha+1)} C_{f}\right)\|x\| \\
\leq & (1-\beta) r+\beta r=r .
\end{aligned}
$$

Now, for $x, y \in C(J, \mathbb{R})$ and for each $t \in J$, we obtain

$$
\begin{aligned}
& |(\Psi x)(t)-(\Psi y)(t)| \\
& \leq \sum_{k=0}^{n-1} \frac{|t-\theta|^{k}}{k !}\left(C_{k}\left(\theta-t_{0}\right)\|x-y\|+\frac{\left(\theta-t_{0}\right)^{\alpha-k}}{\Gamma(\alpha-k+1)} C_{f}\|x-y\|\right) \\
& \quad+\frac{\left(t-t_{0}\right)^{\alpha}}{\Gamma(\alpha+1)} C_{f}\|x-y\| \\
& \leq\left(\sum_{k=0}^{n-1} \frac{|T-\theta|^{k}}{k !}\left(C_{k}\left(\theta-t_{0}\right)+\frac{\left(\theta-t_{0}\right)^{\alpha-k}}{\Gamma(\alpha-k+1)} C_{f}\right)+\frac{\left(T-t_{0}\right)^{\alpha}}{\Gamma(\alpha+1)} C_{f}\right)\|x-y\| \\
& \leq \beta\|x-y\|,
\end{aligned}
$$

where $\beta$ is given by (4.3). As $\beta<1, \Psi$ is a contraction. Thus, the conclusion of the theorem follows by the contraction mapping principle. This completes the proof.

Theorem 4.5 Let $f, g_{k}: J \times \mathbb{R} \rightarrow \mathbb{R}(k=0,1, \ldots, n-1)$ be continuous functions and let there exist positive constants $D_{f}, E_{f}, D_{k}, E_{k}, M$, and $N$ such that

$$
|f(t, x)| \leq D_{f}|x|+E_{f}, \quad\left|g_{k}(t, x)\right| \leq D_{k}|x|+E_{k}, \quad \forall t \in J, x \in \mathbb{R},
$$




$$
\begin{aligned}
& M=\frac{E_{f}\left(T-t_{0}\right)^{\alpha}}{\Gamma(\alpha+1)}+\sum_{k=0}^{n-1} \frac{|T-\theta|^{k}}{k !}\left(\left|b_{k}\right|+\left(\theta-t_{0}\right) E_{k}+\frac{E_{f}\left(\theta-t_{0}\right)^{\alpha-k}}{\Gamma(\alpha-k+1)}\right)>0, \\
& N=\frac{D_{f}\left(T-t_{0}\right)^{\alpha}}{\Gamma(\alpha+1)}+\sum_{k=0}^{n-1} \frac{|T-\theta|^{k}}{k !}\left(\left(\theta-t_{0}\right) D_{k}+\frac{D_{f}\left(\theta-t_{0}\right)^{\alpha-k}}{\Gamma(\alpha-k+1)}\right)<1 .
\end{aligned}
$$

Then the problem (1.1) has at least one solution on J.

Proof Define a suitable ball $B_{R} \subset C(J, \mathbb{R})$ with radius $R>0$ as $B_{R}=\{x \in C(J, \mathbb{R}):\|x\|<R\}$, where $R$ will be fixed later. Then it is sufficient to show that $\Psi: \bar{B}_{R} \rightarrow C(J, \mathbb{R})$ satisfies

$$
0 \notin(I-\lambda \Psi)\left(\partial B_{R}\right),
$$

for any $x \in \partial B_{R}$, and $\lambda \in[0,1]$. Define the homotopy

$$
h_{\lambda}(x)=H(\lambda, x)=x-\lambda \Psi x, \quad x \in C(J, \mathbb{R}), \lambda \in[0,1] .
$$

Then, by the Arzela-Ascoli theorem, $h_{\lambda}$ is completely continuous. If (4.4) is true, then the Leray-Schauder degrees are well defined. Let $I$ denote the identity operator. Then the homotopy invariance and normalization properties of topological degrees imply that

$$
\begin{aligned}
\operatorname{deg}\left(h_{\lambda}, B_{R}, 0\right) & =\operatorname{deg}\left((I-\lambda \Psi), B_{R}, 0\right)=\operatorname{deg}\left(h_{1}, B_{R}, 0\right) \\
& =\operatorname{deg}\left(h_{0}, B_{R}, 0\right)=\operatorname{deg}\left(I, B_{R}, 0\right)=1,
\end{aligned}
$$

since $0 \in B_{R}$. By the nonzero property of the Leray-Schauder degree, $h_{1}(x)=x-\Psi x=0$ for at least one $x \in B_{R}$. In order to find $R$, we assume that $x(t)=\lambda \Psi x(t)$ for some $\lambda \in[0,1]$ and for all $t \in J$. Then

$$
\begin{aligned}
|x(t)| & |\lambda \Psi x(t)| \leq \int_{t_{0}}^{t} \frac{(t-s)^{\alpha-1}}{\Gamma(\alpha)}|f(s, x(s))| d s \\
& +\sum_{k=0}^{n-1} \frac{(t-\theta)^{k}}{k !}\left(\left|b_{k}\right|+\int_{t_{0}}^{\theta}\left|g_{k}(s, x(s))\right| d s+\int_{t_{0}}^{\theta} \frac{(\theta-s)^{\alpha-k-1}}{\Gamma(\alpha-k)}|f(s, x(s))| d s\right) \\
\leq & \sum_{k=0}^{n-1} \frac{|t-\theta|^{k}}{k !}\left(\left|b_{k}\right|+\left(\theta-t_{0}\right)\left(D_{k}\|x\|+E_{k}\right)+\frac{\left(\theta-t_{0}\right)^{\alpha-k}}{\Gamma(\alpha-k+1)}\left(D_{f}\|x\|+E_{f}\right)\right) \\
& +\frac{\left(t-t_{0}\right)^{\alpha}}{\Gamma(\alpha+1)}\left(D_{f}\|x\|+E_{f}\right) \\
\leq & \frac{E_{f}\left(t-t_{0}\right)^{\alpha}}{\Gamma(\alpha+1)}+\sum_{k=0}^{n-1} \frac{|t-\theta|^{k}}{k !}\left(\left|b_{k}\right|+\left(\theta-t_{0}\right) E_{k}+\frac{E_{f}\left(\theta-t_{0}\right)^{\alpha-k}}{\Gamma(\alpha-k+1)}\right) \\
& +\left(\frac{D_{f}\left(t-t_{0}\right)^{\alpha}}{\Gamma(\alpha+1)}+\sum_{k=0}^{n-1} \frac{|t-\theta|^{k}}{k !}\left(\left(\theta-t_{0}\right) D_{k}+\frac{D_{f}\left(\theta-t_{0}\right)^{\alpha-k}}{\Gamma(\alpha-k+1)}\right)\right)\|x\| \\
\leq & M+N\|x\|,
\end{aligned}
$$


which, after taking the supremum norm and solving for $\|x\|$, yields

$$
\|x\| \leq \frac{M}{1-N}
$$

Letting $R=\frac{M-N+1}{1-N},(4.4)$ holds. This completes the proof.

Remark 4.6 Following the method of proof employed in this section, we can obtain the existence results for nonlinear variants of problems (3.8) and (3.9).

Remark 4.7 (Special cases) We obtain the existence results for an initial value problem with initial conditions: $x^{(k)}(\theta)=b_{k}, k=0,1,2, \ldots, n-1$ by taking $\theta=t_{0}$ in the results of this paper. In this case, the operator given by (4.2) takes the form

$$
\Psi x(t)=\sum_{k=0}^{n-1} \frac{\left(t-t_{0}\right)^{k}}{k !} b_{k}+\frac{1}{\Gamma(\alpha)} \int_{t_{0}}^{t}(t-s)^{\alpha-1} f(s, x(s)) d s .
$$

Further, our results correspond to the ones for a problem with classical nonlinear integral conditions:

$$
x^{(k)}(T)=b_{k}+\int_{t_{0}}^{T} g_{k}(s, x(s)) d s, \quad k=0,1,2, \ldots, n-1,
$$

if we fix $\theta=T$ in the obtained results.

Example 4.8 Consider the following nonlinear fractional boundary value problem:

$$
\left\{\begin{array}{l}
{ }^{C} D_{0}^{\sqrt{10}} x(t)=\frac{t|x(t)|}{16(t+|x(t)|)}+\frac{7}{16}, \quad t \in[0,1], \\
x^{(k)}(0.5)=1+\int_{0}^{0.5} \frac{s^{k}}{2(k+1)} \sin \left(\frac{x(s)}{2}\right) d s, \quad k=0,1,2,3 .
\end{array}\right.
$$

Here $\alpha=\sqrt{10}, \theta=0.5, b_{k}=1, f(t, x(t))=\frac{t|x(t)|}{16(1+|x(t)|)}+\frac{7}{16}$, and $g_{k}(t, x(t))=\frac{t^{k}}{2(k+1)} \sin \left(\frac{x(t)}{2}\right)$. With the given values, it is found that $\mu_{f}(t)=\frac{t}{16}+\frac{7}{16}, \mu_{k}(t)=\frac{t^{k}}{2(k+1)}$ with $\left\|\mu_{f}\right\|=\frac{1}{2},\left\|\mu_{k}\right\|=\frac{1}{2(k+1)}$, $k=0,1,2,3$, and

$$
\eta=\sum_{k=0}^{3} \frac{1}{k ! 2^{k}}\left[\frac{1}{4(k+1)}+\frac{\left(\frac{1}{2}\right)^{\sqrt{10}-k}}{16 \Gamma(\sqrt{10}-k+1)}\right] \simeq 0.333942<1
$$

that is, the assumption $\left(\mathrm{A}_{2}\right)$ of Theorem 4.3 is satisfied. Thus, all the conditions of Theorem 4.3 are satisfied. Hence the problem (4.5) has a solution on [0,1]. Also $\beta$ given by (4.3) is such that $\beta \simeq 0.342407<1$. This suggests that the problem (4.5) has a unique solution by the conclusion of Theorem 4.4.

Competing interests

The authors declare that they have no competing interests. 


\section{Author details}

'NAAM-Research Group, Department of Mathematics, Faculty of Science, King Abdulaziz University, P.O. Box 80203, Jeddah, 21589, Saudi Arabia. ${ }^{2}$ Department of Mathematics, Al-Azhar University-Gaza, P.O. Box 1277, Gaza Strip, Palestine. ${ }^{3}$ Department of Mathematics, Texas A \& M University, Kingsville, TX 78363-8202, USA.

\section{Acknowledgements}

The authors thank the reviewers for their useful comments, which led to the improvement of the original manuscript. The paper was funded by the Deanship of Scientific Research (DSR), King Abdulaziz University. The authors, therefore, acknowledge with thanks DSR technical and financial support.

\section{Received: 13 August 2015 Accepted: 12 November 2015 Published online: 01 December 2015}

\section{References}

1. Meral, F, Royston, T, Magin, R: Fractional calculus in viscoelasticity: an experimental study. Commun. Nonlinear Sci. Numer. Simul. 15, 939-945 (2010)

2. Oldham, K: Reactional differential equations in electrochemistry. Adv. Eng. Softw. 41, 9-12 (2010)

3. Lee, C, Chang, F: Fractional-order PID controller optimization via improved electromagnetism-like algorithm. Expert Syst. Appl. 37, 8871-8878 (2010)

4. Ahmed, E, El-Sayed, A, El-Saka, H: Equilibrium points, stability and numerical solutions of fractional-order predator-prey and rabies models. J. Math. Anal. Appl. 325, 542-553 (2007)

5. Liu, F, Burrage, K: Novel techniques in parameter estimation for fractional dynamical models arising from biological systems. Comput. Math. Appl. 62, 822-833 (2011)

6. Mophou, G: Optimal control of fractional diffusion equation. Comput. Math. Appl. 61, 68-78 (2011)

7. Wang, J, Zhou, Y, Wei, W: Optimal feedback control for semilinear fractional evolution equations in Banach spaces. Syst. Control Lett. 61, 472-476 (2012)

8. Gorenflo, R, Mainardi, F: Some recent advances in theory and simulation of fractional diffusion processes. J. Comput. Appl. Math. 229, 400-415 (2009)

9. Jiang, $\mathrm{X}, \mathrm{Xu}, \mathrm{M}, \mathrm{Qi}, \mathrm{H}$ : The fractional diffusion model with an absorption term and modified Fick's law for non-local transport processes. Nonlinear Anal., Real World Appl. 11, 262-269 (2010)

10. Sokolov, I, Chechkin, A, Klafter, J: Fractional diffusion equation for a power-law truncated Levy process. Physica A 336, 245-251 (2004)

11. Nigmatullin, R, Omay, T, Baleanu, D: On fractional filtering versus conventional filtering in economics. Commun Nonlinear Sci. Numer. Simul. 15, 979-986 (2010)

12. Odibat, Z: A note on phase synchronization in coupled chaotic fractional order systems. Nonlinear Anal., Real World Appl. 13, 779-789 (2012)

13. Agrawal, OP: Generalized variational problems and Euler-Lagrange equations. Comput. Math. Appl. 59, 1852-1864 (2010)

14. Kilbas, AA, Srivastava, HM, Trujillo, Jj: Theory and Applications of Fractional Differential Equations. Elsevier, Amsterdam (2006)

15. Matar, M: Existence and uniqueness of solutions to fractional semilinear mixed Volterra-Fredholm integrodifferential equations with nonlocal conditions. Electron. J. Differ. Equ. 2009, 155 (2009)

16. Agarwal, RP, Ahmad, B: Existence theory for anti-periodic boundary value problems of fractional differential equations and inclusions. Comput. Math. Appl. 62, 1200-1214 (2011)

17. Matar, M: On existence and uniqueness of the mild solution for fractional semilinear integro-differential equations. J. Integral Equ. Appl. 23(3), 1-10 (2011)

18. Bai, ZB, Sun, W: Existence and multiplicity of positive solutions for singular fractional boundary value problems. Comput. Math. Appl. 63, 1369-1381 (2012)

19. Henderson, J, Ouahab, A: A Filippov's theorem, some existence results and the compactness of solution sets of impulsive fractional order differential inclusions. Mediterr. J. Math. 9, 453-485 (2012)

20. Grace, SR, Agarwal, RP, Wong, PJY, Zafer, A: On the oscillation of fractional differential equations. Fract. Calc. Appl. Anal. $15,222-231(2012)$

21. O'Regan, D, Stanek, S: Fractional boundary value problems with singularities in space variables. Nonlinear Dyn. 71, 641-652 (2013)

22. Zhang, L, Ahmad, B, Wang, G, Agarwal, RP: Nonlinear fractional integro-differential equations on unbounded domains in a Banach space. J. Comput. Appl. Math. 249, 51-56 (2013)

23. Graef, JR, Kong, L, Wang, M: Existence and uniqueness of solutions for a fractional boundary value problem on a graph. Fract. Calc. Appl. Anal. 17, 499-510 (2014)

24. Nyamoradi, N: Infinitely many solutions for a class of fractional boundary value problems with Dirichlet boundary conditions. Mediterr. J. Math. 11, 75-87 (2014)

25. Zhang, L, Ahmad, B, Wang, G: Successive iterations for positive extremal solutions of nonlinear fractional differential equations on a half line. Bull. Aust. Math. Soc. 91, 116-128 (2015)

26. Alsaedi, A, Ahmad, B, Kirane, M, Mostefaoui, IM: Maximum principle for certain generalized time and space-fractional diffusion equations. Q. Appl. Math. 73, 163-175 (2015)

27. Ahmad, B, Nieto, J: Riemann-Liouville fractional integro-differential equations with fractional nonlocal integral boundary conditions. Bound. Value Probl. 2011, 36 (2011)

28. Salem, H: Fractional order boundary value problem with integral boundary conditions involving Pettis integral. Acta Math. Sci. 31, 661-672 (2011)

29. Cabada, A, Wang, G: Positive solutions of nonlinear fractional differential equations with integral boundary value conditions. J. Math. Anal. Appl. 389, 403-411 (2012)

30. Lakoud, A, Khaldi, R: Solvability of a fractional boundary value problem with fractional integral condition. Nonlinear Anal. 75, 2692-2700 (2012)

31. Ahmad, B, Agarwal, RP: Some new versions of fractional boundary value problems with slit-strips conditions. Bound. Value Probl. 2014, 175 (2014) 
32. Alsaedi, A, Ntouyas, SK, Agarwal, RP, Ahmad, B: On Caputo type sequential fractional differential equations with nonlocal integral boundary conditions. Adv. Differ. Equ. 2015, 33 (2015)

33. Smart, DR: Fixed Point Theorems. Cambridge University Press, Cambridge (1980)

Submit your manuscript to a SpringerOpen ${ }^{\circ}$ journal and benefit from:

- Convenient online submission

Rigorous peer review

- Immediate publication on acceptance

- Open access: articles freely available online

- High visibility within the field

- Retaining the copyright to your article

Submit your next manuscript at $>$ springeropen.com 